\title{
Reconstructing Archeological Vessels by Fusing Surface Markings and Border Anchor Points on Fragments
}

\author{
Fernand Cohen, Zexi Liu, and Zhongchuan Zhang \\ Electrical and Computer Engineering, Drexel University, Philadelphia, PA 19104, USA \\ fscohen@coe.drexel.edu, \{z154,zz57\}@drexel.edu
}

\begin{abstract}
This paper presents a method to assist in the tedious process of reconstructing ceramic vessels from excavated fragments. The method exploits vessel surface marking information (models) supplied by the archaeologists along with anchor points on the fragment borders for reconstruction. Marking models are based on expert historical knowledge of the period, provenance of the artifact, and site location. The models need not to be identical to the original vessel, but must be within a geometric transformation of it in most of its parts. Marking matching is based on discrete weighted moments. We use anchor points on the fragment borders for the fragments with no markings. Corresponding anchors on different fragments are identified using absolute invariants, from which a rigid transformation is computed allowing the fragments to be virtually mended. For axially symmetric objects, a global constraint induced by the surface of revolution is applied to guarantee global mending consistency.
\end{abstract}

Keywords: 3D Weighted Moment, Mending, Archeological Shards, Ceramic Fragments, Global Constraint, Virtual Reconstruction.

\section{Introduction}

The mending of unearthed archeological ceramic shards to reconstruct vessels that the fragments once formed is currently a tedious and time-consuming process, and yet is a vital step in interpreting, understanding, and preserving cultural heritage. In this paper, we focus on virtually reconstructing/mending ceramic vessel fragments shown in Figure 1. They are scanned using Konica Vivid 910 3D scanner. Our contribution is that we develop a method that makes use of marking vessel models given by the experts and surface makings (of both fragments and marking models) to drive the reconstruction of the vessels from broken fragments via a novel set of weighted discrete moments, coupled with the use of fragment borders to mend them as a jigsaw puzzle game.

\subsection{Related Work}

Computer vision technology has been used to facility the image and document reassembly. Saharan and Singh [1] use the flood fill algorithm to obtain the closed 


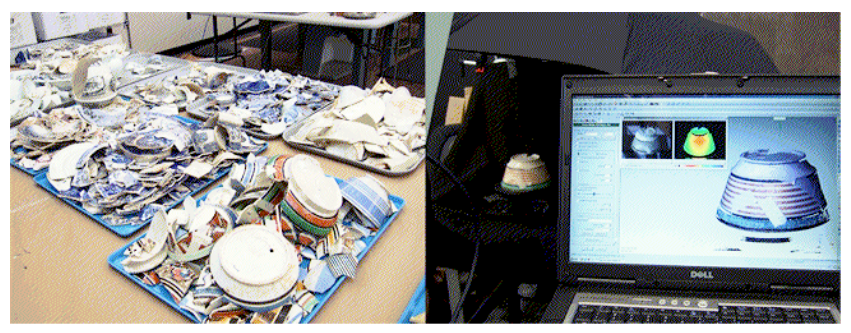

Fig. 1. Ceramic remains and digitized model

boundaries of fragmented images and calculate the local curvature of each boundary pixel which is stored into a string.

The fragment matching is then reduced to a string matching problem. Zhu et al. [2] propose to use turning-function-based partial curve matching to find the candidate matches and define the global consistency as the global criterion to do document reconstruction. The global match confidences are assigned to each candidate match and then these confidences are iteratively updated via the gradient projection method to maximize the criterion. Tsamoura and Pitas [3] instead pre-sent a color based approach to reassemble fragmented images and paintings. A neural network based color quantization approach for the representation of the image contour followed by a dynamic programming technique is employed to identify the matching contour segments of the image fragments. Aminogi et al. [4] utilize both the shape and the color characteristics of the image fragment contours for matching.

\subsection{Marking Models}

Archeologists usually possess a library that consists of excavated broken, unbroken, or mended artifacts, patterns of relief, color markings, and historical documents describing the shape and dimension of various artifacts. The creation of a set of marking models is a process that involves the archaeology experts who are assisted by graphics and computational engineers of rendering in 3D what the archaeology experts perceive and consider as possible representations for possible vessels in a given dig.

A raw template can be one of those unbroken or manually mended vessels. It is scanned and imported into any commercial 3D sculpture software such as Zbrush. When the unbroken/mended vessels are not available, we simply create a 3D template vessel in ZBrush with the information supplied by the archaeologists (e.g., height, neck size, belly size of a vase), and have them interactively modify the template in accordance with what they think are good marking models.

The creation of the marking models is also a dynamic process. If the marking models in one category have very few fragments aligned to them, the archeologists will make changes to the shape, the marking patterns, or the location of these patterns, creating new variations. An overview of the proposed system is shown in Figure 2. 


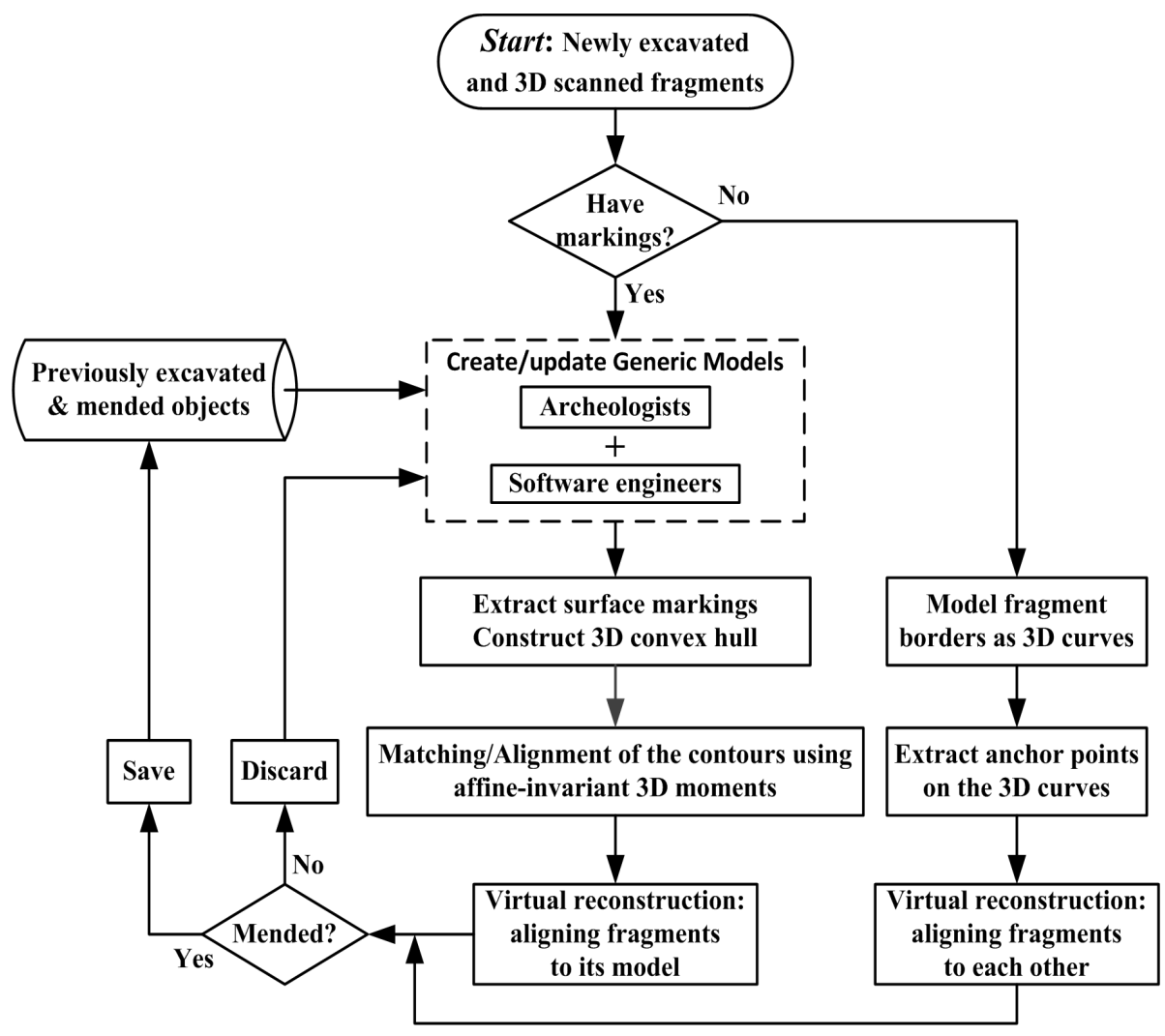

Fig. 2. System overview

\section{Reconstruction of Archeological Vessels}

\subsection{Alignment Using Marking Models}

Figure 3 shows the mending procedure based on the surface markings, the patterns drawn on a vessel and usually having different colors from most other parts of a vessel. The surface markings on the marking models and fragments are first extracted by thresholding the color information of the markings and/or the background. For each surface marking, a 3D convex hull is computed based on its containing points. Corresponding markings are established using the 3D convex hulls, based on which the transformation between a matched marking pairs is computed. The transformation giving the smallest alignment error is used to align a fragment to the marking model. 


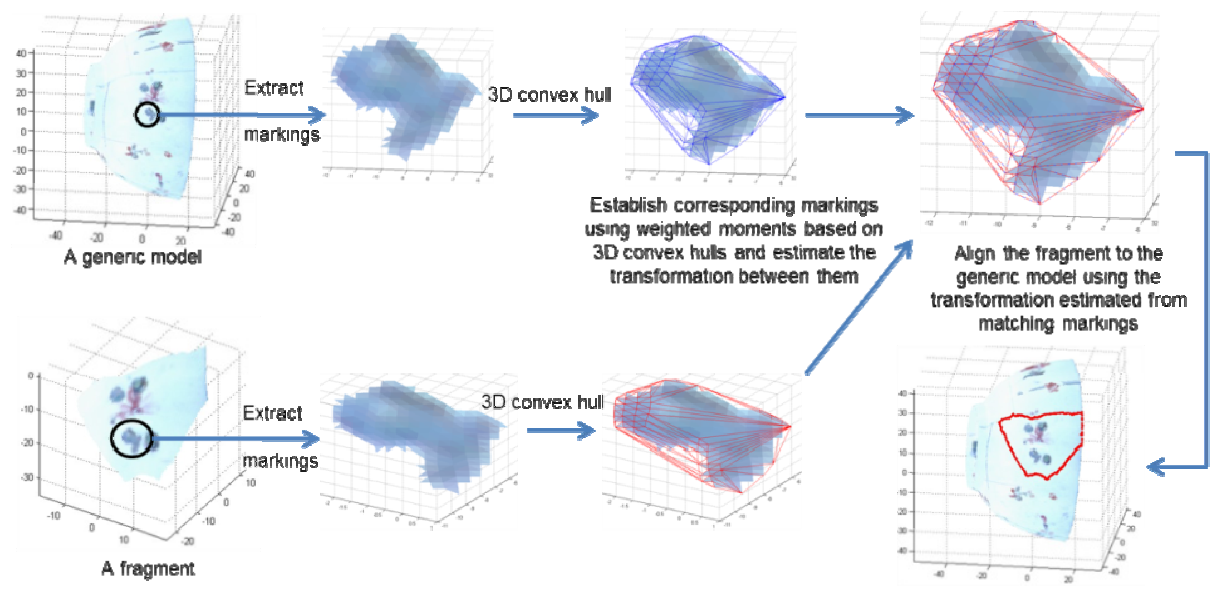

Fig. 3. Corresponding markings and their convex hull

\subsubsection{Weighted Moments}

With a data set $R=\left\{\boldsymbol{r}_{\boldsymbol{i}}=\left(x_{i}, y_{i}, z_{i}\right) \mid i=1,2, \ldots, n\right\}$, and an s-weighted tion $w\left(\boldsymbol{r}_{\boldsymbol{i}}, \boldsymbol{r}_{\boldsymbol{j}}, \boldsymbol{r}_{\boldsymbol{k}}\right)_{s}$, the s-weighted central moment is defined as $\mu(a, b, c)_{s}=$ $\sum_{i=1}^{n}\left(x_{i}-\bar{x}\right)^{a}\left(y_{i}-\bar{y}\right)^{b}\left(z_{i}-\bar{z}\right)^{c} \sum_{j=1}^{n} \sum_{k=1}^{n} w\left(\boldsymbol{r}_{\boldsymbol{i}}, \boldsymbol{r}_{\boldsymbol{j}}, \boldsymbol{r}_{\boldsymbol{k}}\right)_{s}$, where $(\bar{x}, \bar{y}, \bar{z})$ is the center of the data set. The choice of the weight function is dictated by the relative affine invariant which allows for the moments to factor linearly in the affine parameters.

\subsubsection{Declaring Corresponding Markings on Fragment and Vessel}

As the number of convex hull vertices is generally much less than the marking points, the computation load of the weighted moments is reduced when using the convex hull as a compressed representation of a surface marking. Since the volume of a convex hull is a relative invariant under the affine transformation $T=\{[L], \boldsymbol{B}\}$, the s-weighted function is defined as $w\left(\boldsymbol{r}_{\boldsymbol{i}}, \boldsymbol{r}_{\boldsymbol{j}}, \boldsymbol{r}_{\boldsymbol{k}}\right)_{s}=\left|\left(\boldsymbol{r}_{\boldsymbol{i}}-\boldsymbol{r}_{\mathbf{0}}\right) \cdot\left[\left(\boldsymbol{r}_{\boldsymbol{j}}-\boldsymbol{r}_{\mathbf{0}}\right) \times\left(\boldsymbol{r}_{\boldsymbol{k}}-\boldsymbol{r}_{\mathbf{0}}\right)\right]\right|^{s}$, where $\boldsymbol{r}_{\boldsymbol{i}}, \boldsymbol{r}_{\boldsymbol{j}}$ and $\boldsymbol{r}_{\boldsymbol{k}}$ are 3 vertices on the convex hull, and $\boldsymbol{r}_{\mathbf{0}}$ is the centroid. If $\boldsymbol{r}_{\boldsymbol{a} \boldsymbol{i}}$ is the affine counterpart of $\boldsymbol{r}_{\boldsymbol{i}}$ after the transform $T$, we have $\boldsymbol{r}_{\boldsymbol{a} i}=[L] \boldsymbol{r}_{\boldsymbol{i}}+\boldsymbol{B}$. The zero ${ }^{\text {th }}$ order s-weighted affine invariant central moments are related by $\mu_{a}(0,0,0)_{s}=$ $|\operatorname{det}\{[L]\}|^{s} \mu(0,0,0)_{s}$, where $\mu(0,0,0)_{s}=\sum_{i=1}^{n} \sum_{j=1}^{n} \sum_{k=1}^{n} w\left(\boldsymbol{r}_{\boldsymbol{i}}, \boldsymbol{r}_{\boldsymbol{j}}, \boldsymbol{r}_{\boldsymbol{k}}\right)_{s}$ and $\mu_{a}(0,0,0)_{s}=\sum_{i=1}^{n} \sum_{j=1}^{n} \sum_{k=1}^{n} w_{a}\left(\boldsymbol{r}_{\boldsymbol{a} i}, \boldsymbol{r}_{\boldsymbol{a} \boldsymbol{j}}, \boldsymbol{r}_{\boldsymbol{a} \boldsymbol{k}}\right)_{s}$. Absolute affine invariant (AAI) can be constructed from these relative invariants by using any two different weight factors $s_{0}$ and $s_{1}: \operatorname{AAI}\left(s_{0}, s_{1}\right)=\frac{s_{1}}{\sqrt[s_{0}(0,0,0)_{s_{1}}]{\mu_{a}(0,0,0)_{s_{0}}}}=\frac{\sqrt[s_{1}]{\mu(0,0,0)_{s_{1}}}}{\sqrt{s_{0}}}$, where $\mu_{a}(0,0,0)_{s_{0}}$, $\mu(0,0,0)_{s_{0}} \neq 0$ since $w_{a}\left(\boldsymbol{r}_{\boldsymbol{a} \boldsymbol{i}}, \boldsymbol{r}_{\boldsymbol{a} \boldsymbol{j}}, \boldsymbol{r}_{\boldsymbol{a} \boldsymbol{k}}\right)_{s}, w\left(\boldsymbol{r}_{\boldsymbol{i}}, \boldsymbol{r}_{\boldsymbol{j}}, \boldsymbol{r}_{\boldsymbol{k}}\right)_{s}>0$. The AAI is used to establish which convex hull of marking on the marking models corresponds to a given one on the fragment. Corresponding convex hulls (not markings) are declared if their AAIs are close enough. Once the convex hull correspondences are established, the 


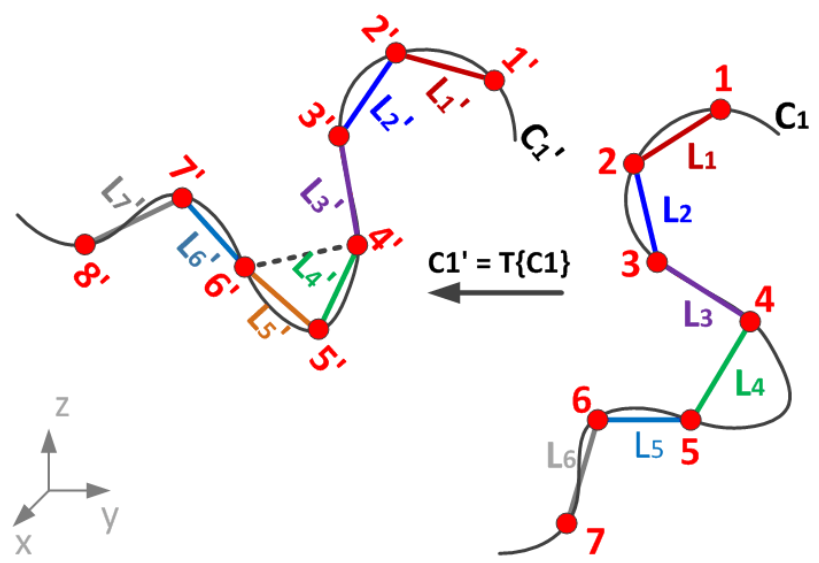

Fig. 4. Absolute invariants with anchor points with some possible missing. The algorithm in section 2.2.2 will always find the longest matching string.

affine transformation that maps the convex hull on the fragment onto its corresponding one on the vessel is computed as explained in 2.1.3.

\subsubsection{Aligning Fragments against Marking Models}

Given corresponding convex hull pairs determined using AAIs, we proceed to estimate the unique affine transformation $T=\{[L], B\}$ that will align them. Towards that end, we use a set of first order s-weighted affine invariant central moments. To solve the transformation $[L]$ that has 9 unknowns, a set of 3 different $s$ values is used to arrive at 9 equations with 9 unknowns. This results in the linear equation

$$
\left[M_{a}\right]=[L][M]=[L]\left[\begin{array}{lll}
\frac{\mu(1,0,0)_{s 1}}{\mu(0,0,0)_{s 1}} & \frac{\mu(1,0,0)_{s 2}}{\mu(0,0,0)_{s 2}} & \frac{\mu(1,0,0)_{s 3}}{\mu(0,0,0)_{s 3}} \\
\frac{\mu(0,1,0)_{s 1}}{\mu(0,0,0)_{s 1}} & \frac{\mu(0,1,0)_{s 2}}{\mu(0,0,0)_{s 2}} & \frac{\mu(0,1,0)_{s 3}}{\mu(0,0,0)_{s 3}} \\
\frac{\mu(0,0,1)_{s 1}}{\mu(0,0,0)_{s 1}} & \frac{\mu(0,0,1)_{s 2}}{\mu(0,0,0)_{s 2}} & \frac{\mu(0,0,1)_{s 3}}{\mu(0,0,0)_{s 3}}
\end{array}\right]
$$

And $\left[M_{a}\right]$ is the counterpart of $[M]$. The transformation $[L]$ is uniquely computed from (1). Once $[L]$ is found, the translation parameters $\boldsymbol{B}$ can be obtained from the centroids of the matched convex hull pair. To evaluate the goodness of the alignment we use the average distance error between all the 3D points (not just those on the marking) on the morphed fragment to their closest points on the vessel. The morphing of the fragment into the vessel coordinate space is done in accordance with the estimated affine transformation $T$. 

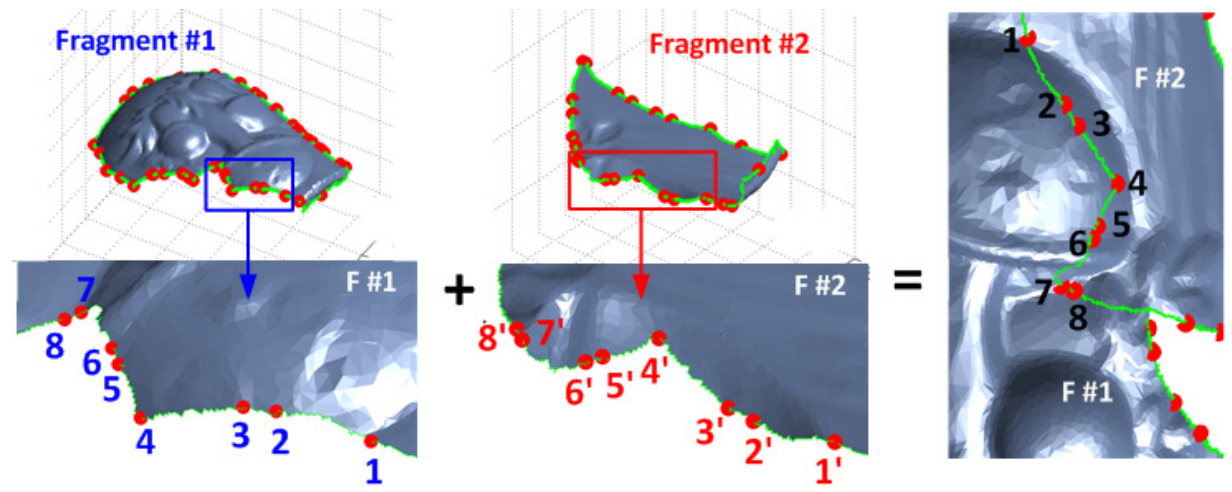

Fig. 5. Fragment mending based on anchor points (red dots)

\subsection{Mending Fragments Using Anchor Points on Borders}

When the fragments have no markings on them or if we want to use complementary information for mending, landmarks on fragment borders are used. The rigid transformation preserved landmarks used in this paper are inflection, corner, and zerotorsion points.

\subsubsection{Constructing Absolute Invariants Using Anchor Points}

Since length is preserved under 3D rigid maps and are absolute invariants, we can construct a sequence of absolute invariants by considering the sequence of length of lines between the two consecutive anchor points (e.g., lines between points \#1-2, points \#2-3, etc. as shown in Figure 4).

Although an anchor point is missing between points \#4 and \#5 on curve $C_{1}$, the matching is not affected. For a curve with $n$ anchor points, the sequence of length is denoted by $I=\left(L_{1}, L_{2}, \ldots, L_{n-1}\right)$. The transformed lines sequence has also the same number of elements $I^{\prime}=\left(L_{1}^{\prime}, L_{2}^{\prime}, \ldots, L_{n-1}^{\prime}\right)$. And we need to find the sequences of elements in that invariant vectors in $I$ and $I^{\prime}$ that correspond. Towards that end we introduce the "longest string search" technique, which is similar to the "list-matching algorithm" [5] for establishing the correspondence and declaring the match. To recover the rigid transformation at least 3 pairs of matched points is required, i.e., the minimum edge string length should be 2 .

\subsubsection{Longest String Match between Fragments}

A set of two or more consecutive anchor points and their corresponding invariants are considered to be a "string" (e.g., the set $(i, i+1, i+2, \ldots)$ on one fragment and the set $(j, j+1, j+2, \ldots)$ on another fragment). We allow for a small error $(2-5 \%)$ in the values of the invariants when declaring matching edges. i.e., the sets $(i, i+1, i+$ $2, \ldots)$ and $(j, j+1, j+2, \ldots)$ of anchor points are declared as matching if $\mid I^{\prime}(j)-$ $I(i) \mid<0.05 I(i)$ for every pair in the two sets. In case that there are missing points, 

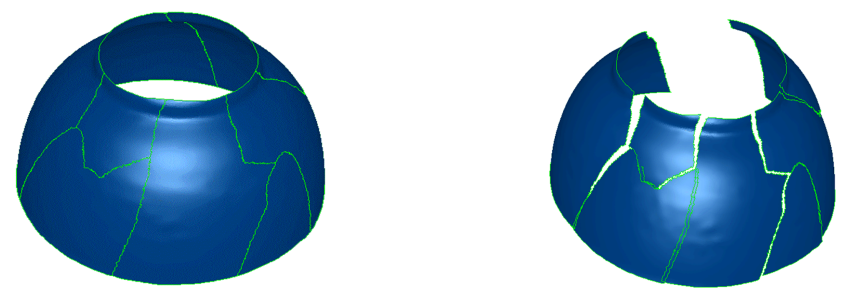

Fig. 6. With/without global optimization

for example, in Figure $4, L_{4} \neq L_{4^{\prime}}$, then the distance $L_{5^{\prime}}$ will be discarded and the distance between points $4^{\prime}$ and $6^{\prime}$ will be calculated, then $L_{4}=L_{4^{\prime} 6^{\prime}}$. Generally speaking, if we encounter an "unmatched" segment, we will always jump over one point and check its next anchor point. As this process is recursive we can deal with more than one missing point. The rigid transformation $T=\{[R], t\}$ can be recovered from three pairs of matched points, or estimated from more matched anchor points using a least square error (LSE) estimation method [6]. Once the transformation parameters are found, the fragments are mapped into the same coordinates system by undoing the rigid transformation and the fragments are aligned.

\subsubsection{Global Optimization}

The mending described in the previous section and shown in Figure 5 is a pair wise mending process. It is conceivable that alignment errors will accumulate, rendering the reconstruction result less than satisfactory (See right hand side of Figure 6).

For vessels that are axially symmetric, this problem can be solved by adopting the surface of revolution as a global constraint. The surface of revolution is obtained by going through the following steps: 1) Extract the "profile curve" [7]; 2) Obtain the symmetry axis (revolution axis); 3) Rotate the profile curve about the axis; 4) Generate a rotation surface; 5) Use the surface as a global optimization "basis". Steps 1 2 are shown in Figure 7 left.

The lower rim and upper rim are obtained by fitting a circle to the fragment border segments. Of all the fragment border segments, the one with the smallest fitting error is the rim. The cross-section curve $c$ is the profile curve. Steps 3 4 are shown in Figure 7 right. Here we rotate the profile curve about the revolution axis to generate a set of curves which constitute the rotation surface. This rotation surface is used in our mending process as a basis shape, where the fragments are not only aligned to each
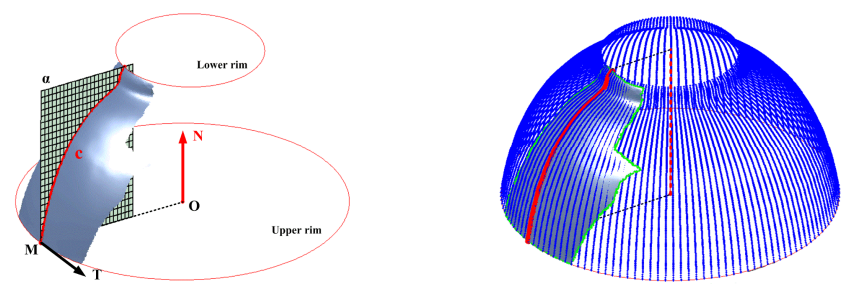

Fig. 7. Obtain profile curve, symmetric axis and generate a rotation surface 

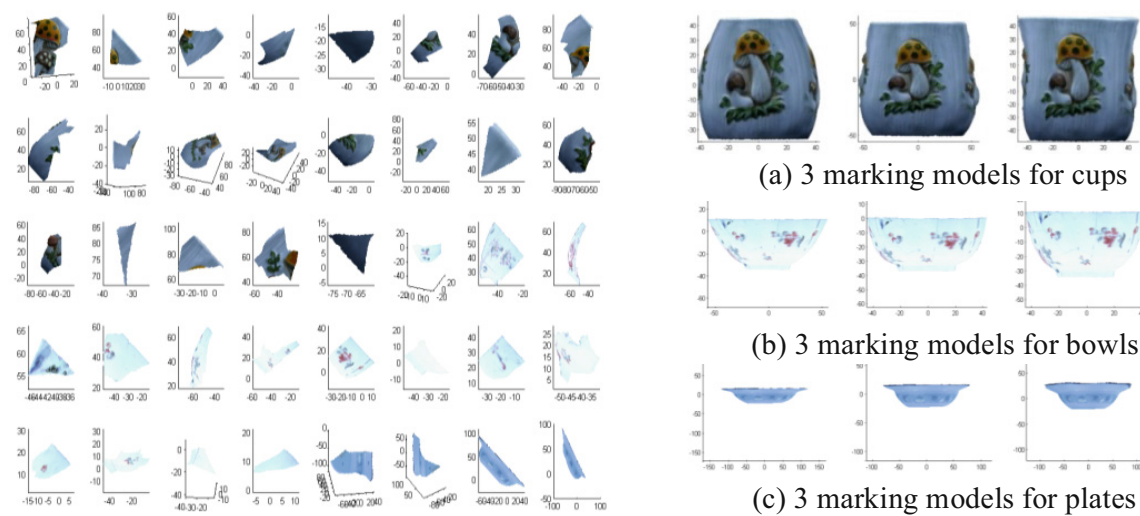

(a) 3 marking models for cups
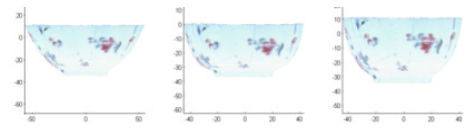

(b) 3 marking models for bowls

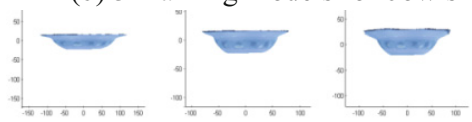

(c) 3 marking models for plates

Fig. 8. 3D scanned fragments and marking models

other, but are also aligned to this surface. With this global optimization constraint, the result is improved as shown on the left hand side of Figure 6. Note that before a basis shape is found, fragments with no upper and lower rims are set aside, and only fragments that do possess both rims are found and possibly mended using the pair wise invariants approach. After we obtain a mended fragment with both upper and lower rims, we extract the profile curve and generate the rotation surface (steps 1-5). We then improve on the mending for all fragments using the basis shape as a global constraint.

\section{Experiments}

We test our methods on 62 fragments (40 of them have markings and are shown in Figure 8 left. The 22 fragments with no markings are mended later using the anchor point method. The fragments are from 3 types of vessels: cups, bowls and plates. The archaeologists provide 20 marking models for each type of vessel, hence a total of 60 possible marking models. We show 3 marking models for each category (cups, bowls and plates) in Figure 8 right.

The reconstruction results of the 62 fragments are shown in Figure 9. The fragments bounded by red boundaries are aligned using markings and marking models.

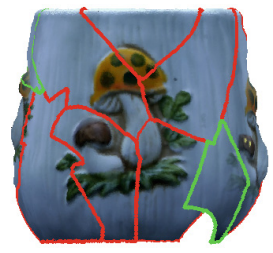

(a)

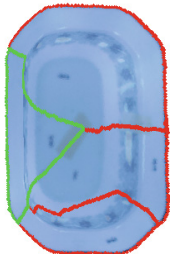

(b)

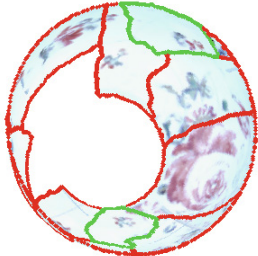

(c)

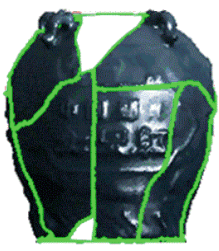

(d)

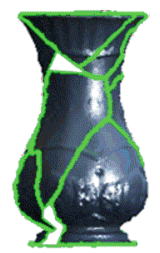

(e)

Fig. 9. Reconstruction results 
The fragments bounded by green boundaries are mended using anchor points. We find that most fragments can be aligned against one marking model for each category of vessels. However, as the marking models are not exact, it's possible that parts of the best marking model are not close enough to the true vessel, which resulted in having a few fragments not aligned with the best marking model. Based on markings alone, $70 \%$ of fragments with markings (red boundaries in Figure 9) are aligned to the best models. This figure changes upward to around $90 \%$ if we consider the markings along with the anchor points in the mending. Finally, out of the 22 fragments that don't have any markings on, $18(>80 \%)$ fragments are properly mended (see Figure 9 (d), (e)). The remaining 4 fragments had insufficient number of anchor points on their borders and hence couldn't be mended. In the end, 54 (>85\%) out of the 62 fragments are successfully mended, when using markings plus anchor points for the first set and anchor points for the set with no markings on them.

\section{Conclusions}

We present novel complementary methods to reconstruct vessels virtually by aligning 3D scanned fragments against vessels based on surface markings and anchor points on borders. These methods weigh between expert opinion (with expected uncertainties), and total lack of prior knowledge. Building in uncertainties in the markings models allow for rotation, scaling, shifting, and shearing between markings on the marking vessels and their corresponding ones on the fragments. Whereas using anchors points as complementary information on fragment surfaces allows for a natural mending when expert prior information is lacking or as cross validation method. This paper shows the importance of using complementary information in the mending process for assisting in the tedious process of vessels reconstruction from fragments.

\section{References}

1. Saharan, R., Singh, C.V.: Reassembly of 2D fragments in image reconstruction. International Journal of Computer Applications 19, 41-45 (2011)

2. Zhu, L., Zhou, Z., Hu, D.: Globally consistent reconstruction of ripped-up documents. IEEE Transactions on Pattern Analysis and Machine Intelligence 30, 1-13 (2008)

3. Tsamoura, E., Pitas, I.: Automatic color based reassembly of fragmented images and paintings. IEEE Transactions on Image Processing 19, 680-690 (2010)

4. Amigoni, F., Gazzani, S., Podico, S.: A method for reassembling fragments in image reconstruction. Presented at the Proceedings of International Conference on Image Processing (2003)

5. Bratko, I.: Prolog Programming for Artificial Intelligence. Addison Wesley, London (1990)

6. Umeyama, S.: Least-Square Estimation of Transformation Parameters Between Two Point Patterns. IEEE Transactions on Pattern Analysis and Machine Intelligence 13, 376-380 (1991)

7. Willis, A., Orriols, X., Cooper, D.B.: Accurately Estimating Sherd 3D Surface Geometry with Application to Pot Reconstruction. In: CVPR Workshop: ACVA, Madison, WI, USA (2003) 